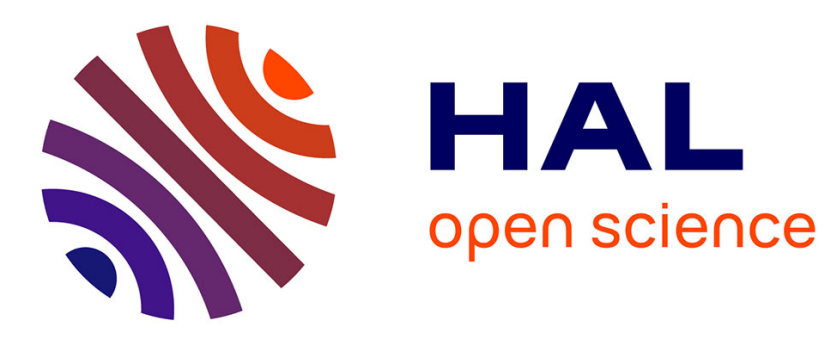

\title{
Requalification des ouvrages anciens. Les ponts en maçonnerie
}

\author{
Nathalie Domede, Gérard Pons, Alain Sellier
}

\section{To cite this version:}

Nathalie Domede, Gérard Pons, Alain Sellier. Requalification des ouvrages anciens. Les ponts en maçonnerie. Revue Européenne de Génie Civil, 2011, 10.1080/17747120.2007.9692984 . hal02155712

\section{HAL Id: hal-02155712 \\ https://hal.science/hal-02155712}

Submitted on 13 Jun 2019

HAL is a multi-disciplinary open access archive for the deposit and dissemination of scientific research documents, whether they are published or not. The documents may come from teaching and research institutions in France or abroad, or from public or private research centers.
L'archive ouverte pluridisciplinaire HAL, est destinée au dépôt et à la diffusion de documents scientifiques de niveau recherche, publiés ou non, émanant des établissements d'enseignement et de recherche français ou étrangers, des laboratoires publics ou privés. 


\title{
Requalification des ouvrages anciens Les ponts en maçonnerie
}

\author{
Nathalie Domede - Gérard Pons - Alain Sellier \\ Laboratoire Matériaux et Durabilité des Constructions, INSA, UPS, 135 avenue de Rangueil \\ 31077 Toulouse CEDEX 4
}

\section{RÉSUMÉ}

L'objectif de la recherche présentée ici est de développer une méthode de requalification des ponts voûtes en maçonnerie par le calcul. La méthode a été mise en œuvre sur une voûte surbaissée construite en 1870 dans le Sud-Ouest de la France. Elle est divisée en trois étapes. La première étape est une recherche historique nécessaire pour connaître de façon précise la constitution de l'ouvrage (géométrie, matériaux, procédures de construction). La seconde étape est expérimentale. Elle a pour but de déterminer la loi de comportement homogénéisée des maçonneries constitutives de l'ouvrage, et une loi d'interface entre les blocs homogénéisés incluant le comportement en traction et en cisaillement des joints de mortier. La troisième étape est le calcul de l'ouvrage, en 3D, à l'aide de la méthode aux éléments finis. L'originalité du modèle est d'intégrer la loi d'interface dans les « éléments joints » et la loi des blocs homogénéisés dans les éléments finis massifs. Une étude paramétrique a permis de déceler les facteurs influençant la sécurité du pont.

\section{ABSTRACT}

The aim of this research is to build an assessment method for masonry arch bridges by modelling and computing. The method was developed and tried on a vault build in 1870 in south-west of France. It is made of three steps. The first one is an historic research. It is necessary to know the exact bridge constitution (geometry, materials, construction process). The second step is an experimental approach whose aim is to establish the homogenized masonries mechanic behaviour law and an interface law between homogenized blocks and including the mortar tensile and shear behaviour. The third step is the 3D computing of the bridge, using a finite element method. The specificity of the model is to take into account the interface law using "joint elements" and the homogenized behaviour law of blocks as a constitutive law of finite elements. A parametric study has established the factors involved in the bridge security.

MOTS-CLÉS : requalification, maçonnerie, voûtes, mortier de chaux, briques, éléments finis, éléments joints.

KEYWORDS: assessment, masonry, arch bridges, lime mortar, bricks, FEM, DEM.

\section{INTRODUCTION}

Pour les maitres d'ouvrage gestionnaires d'un parc d'ouvrages d'art ancien, la maintenance des ponts en maçonnerie est une nécessité. Face à des besoins évolutifs, il ne s'agit pas seulement de " 
conservation » mais aussi de " requalification » du patrimoine, dont le but est de s'assurer de l'aptitude au service d'ouvrages existants et vieillissants compte tenu de l'évolution des sollicitations (augmentation du trafic, véhicules plus rapides et plus lourds). Le « re-calcul » des ponts voûtes s'inscrit dans cette démarche.

Rappelons brièvement les outils de calcul dont les ingénieurs disposent, et qui sont utilisés aujourd'hui. La méthode publiée par Méry en 1830 (décrite notamment par Séjourné en 1913), est une méthode graphique qui consiste à vérifier qu'il existe un équilibre possible des forces dans la voûte. Elle ne donne pas les contraintes réellement atteintes. Le programme VOUTE, développé par J-M Delbecq en 1980 pour le compte du SETRA (SETRA/LCPC, 1982), ainsi que le programme RING, développé récemment par l'Université de Sheffield (Gilbert, 2005), effectuent un calcul à la rupture, sans connaître la loi de comportement des matériaux. Ces deux programmes recherchent l'équilibre limite du pont, en $2 \mathrm{D}$ et calculent la charge de rupture. Ces outils ont en commun les hypothèses suivantes : la voûte est le seul élément résistant du pont, elle est bi-encastrée, les actions sont des charges gravitaires. Il s'agit, dans tous les cas, d'un calcul plan (2D), qui s'affranchit de la connaissance des lois de comportement mécanique des matériaux de la voûte.

Les ingénieurs peuvent également se lancer dans un calcul par la méthode des éléments finis (MEF) avec des logiciels commerciaux. Ces logiciels sont, en général, limités aux matériaux typiques du génie civil : acier, béton, bois. De plus, ils n'intègrent généralement les non linéarités du comportement de ces matériaux que via l'adoption d'un comportement élasto-plastique. La question qui se pose au calculateur est alors de choisir les paramètres mécaniques pour adapter l'outil à la maçonnerie (le module d'élasticité, la résistance à la compression et à la traction).

Très peu de recherches universitaires ont été publiées dans le domaine du calcul des ponts voûtes en trois dimensions. Citons les travaux de T. Boothby et P. Fanning (2001) qui ont utilisé la MEF pour calculer des ponts en pierre d'Irlande. L'intérêt de cette recherche est d'avoir prouvé la complexité des phénomènes internes transversaux, et la participation de toutes les parties de l'ouvrage à la stabilité globale. Cependant, le choix de la loi de comportement des maçonneries, fait par les auteurs, n'est justifié qu'à posteriori par comparaison avec les résultats d'essais en vrai grandeur réalisés sur le pont. Il s'agit alors d'une analyse inverse cherchant la loi de comportement homogène locale pour modéliser au mieux les déplacements du pont. Cette démarche ne fait pas appel à une identification des lois de comportement sur des échantillons des matériaux constitutifs. Elle ne considère donc pas la complexité du comportement local hétérogène de la maçonnerie.

Rappelons néanmoins que la prise en compte du caractère hétérogène des maçonneries a été considérée par quelques auteurs sur des problèmes bidimensionnels. Combescure a proposé d'utiliser une loi élastoplastique homogénéisé (Combescure, 1996). Cruz Diaz et al. ont notamment intégré dans un modèle bidimensionnel de mur un comportement anisotrope endommageable associé à des éléments joints (Cruz-Diaz et al., 2002). Sab, enfin, a proposé d'établir des lois de comportement de maçonnerie bidimensionnelles par homogénéisation (Sab, 2003). Les résultats de ces travaux n'ont toutefois pas encore été étendus aux ouvrages d'art de maçonneries anciennes.

Ainsi, il n'existe pas d'outil simple et performant intégrant le comportement réel de toutes les parties de l'ouvrage étudié, en service et jusqu'à la ruine, dans les trois dimensions de l'espace. Le but de la recherche qui est présentée ici est de progresser dans ce domaine.

La méthode exposée comprend trois phases successives. Il s'agit dans un premier temps de rassembler toutes les informations nécessaires sur l'histoire de l'ouvrage et sa composition par une recherche historique. Dans un deuxième temps, les lois de comportements des matériaux élémentaires constitutifs de l'ouvrage sont déterminées expérimentalement. Enfin, dans la troisième phase, un modèle de calcul numérique est construit, en trois dimensions, à l'aide de la méthode des éléments 
finis. Ce qui fait la spécificité de notre modèle est la présence, d'une part, d'éléments finis de maçonnerie dont le comportement est homogénéisé à partir des lois de comportement et de la géométrie des matériaux élémentaires (briques, joints, moellons...), et, d'autre part, d'éléments joints qui restituent la capacité des matériaux à se fissurer en traction et en cisaillement. Nous allons présenter successivement chacune de ces trois phases, dans leurs principes et résultats généraux, sur la base d'une étude de cas : le pont de la Millette (figure 1).

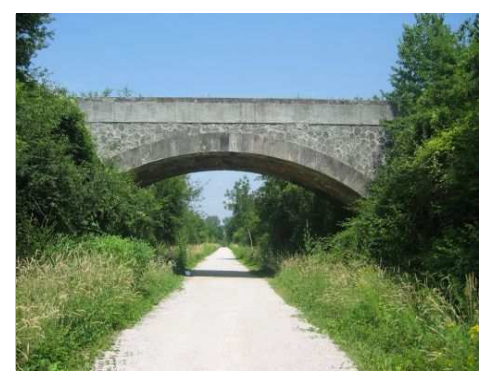

Figure 1. Le pont de la Millette, Tarn (France)

\section{PHASE 1 : RECHERCHE HISTORIQUE}

Dans le cadre de cette recherche, un partenaire a été choisi : Réseau Ferré de France. L'aire d'étude a été restreinte à une ligne de chemin de fer construite en 1870 par la Compagnie des Chemins de fer du Midi, entre Castelnaudary et Albi (Tarn). L'étude des archives disponibles, constituées de plans et de pièces écrites, a abouti à la connaissance précise des ouvrages de la ligne (11 ponts en maçonnerie). Elle a permis la collecte d'informations sur la conception des ponts, leur géométrie externe et interne, les matériaux de construction utilisés, et les moyens de mise en œuvre.

A la lecture des documents conservés, nous observons que la Compagnie des Chemins de fer du Midi effectuait un contrôle rigoureux du chantier et des matériaux mis en œuvre sur les ouvrages en maçonnerie. Ceux-ci constituent un ensemble homogène par la nature et la disposition de leurs matériaux constitutifs. Cette similitude donne un caractère régional à ces ouvrages d'art. Listons les matériaux en présence (figure $2 a$ et $2 b$ ) : corps de voûte en maçonnerie de briques pleines d'Albi, bandeaux latéraux et sommiers en pierre de taille (calcaire de Lautrec), murs tympans en maçonnerie de moellons en opus incertum, remplissage en maçonnerie de moellons bruts. Il faut noter que tous les mortiers sont des mortiers de chaux hydraulique à l'exclusion du ciment (chaufourniers du Tarn).

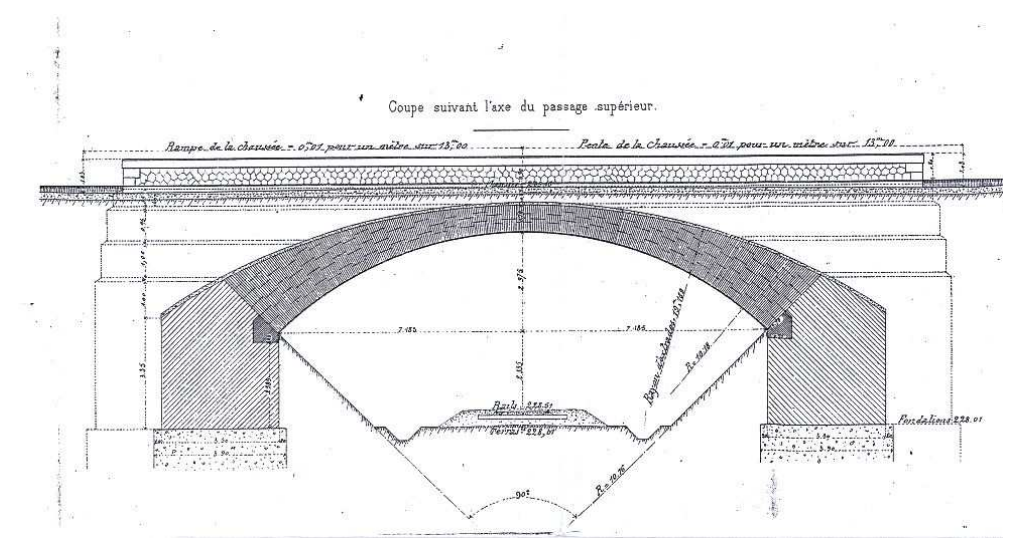

Figure 2a. Plans du pont de la Millette, coupe longitudinale d'après les archives SNCF / RFF, 1870. Ouverture: 14.37m, épaisseur de la voûte en clé : $79 \mathrm{~cm}$, rayon de l'intrados : $10.75 \mathrm{~m}$, largeur hors tout : $4.80 \mathrm{~m}$. 


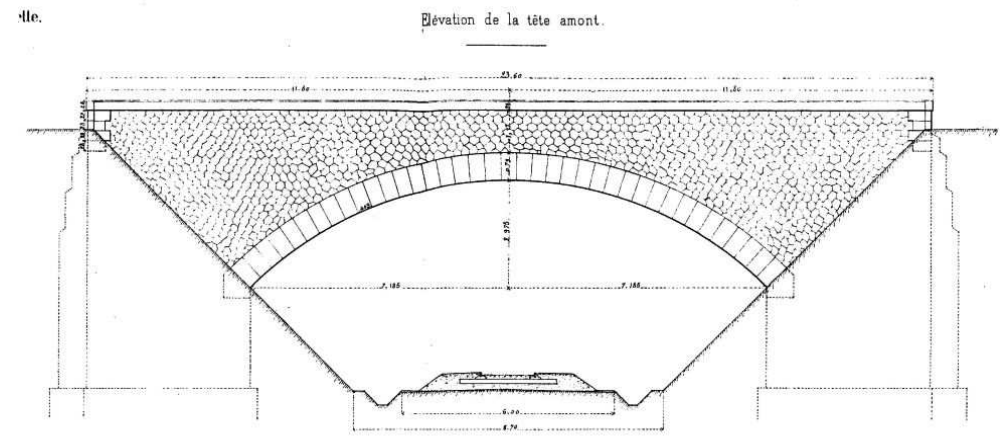

Figure $2 b$. Pont de la Millette, élévation.

\section{PHASE 2 : RECHERCHE EXPERIMENTALE}

Dans cette seconde phase, le but est de déterminer la loi de comportement mécanique des matériaux identifiés par la phase 1. Une étude bibliographique montre qu'il n'existe pas de données suffisantes sur les matériaux anciens, notamment les matériaux à caractère régional tels que la brique pleine de grand format du sud-ouest de la France, et les pierres extraites de petites carrières qui ne sont plus exploitées. Il faut donc procéder à une identification spécifique.

Nous avons réalisé une étude expérimentale afin de déterminer, dans un premier temps, le comportement des matériaux de base (brique, mortier de chaux et pierre calcaire), puis, dans un second temps, celui des maçonneries. Concernant le mortier de chaux, précisons qu'une étude de vieillissement a été réalisée, par carbonatation accélérée, de façon à établir le rapport entre le comportement à 1 mois et le comportement à long terme.

\subsection{Etude et modélisation des matériaux de base}

Une campagne d'essais a été réalisée pour décrire le comportement mécanique des briques, du mortier de chaux et des pierres jusqu'à rupture. Pour ce faire, nous avons travaillé sur des matériaux neufs (en ce qui concerne la brique et la chaux), identiques aux matériaux anciens existants.

Les briques choisies sont des briques pleines de la Société Terre Cuites de Savès, établie sur la commune d'Empeaux en Haute-Garonne. Elles sont moulées mécaniquement, de façon artisanale, non extrudées comme le sont les briques creuses actuelles, puis séchées au séchoir et cuites au four. Leurs dimensions sont $37 \mathrm{~cm} * 24 \mathrm{~cm} * 5 \mathrm{~cm}$. La chaux SOCLI NHL 3.5 a été choisie parce que c'est une chaux hydraulique sans aucun additif. Elle est analogue à une chaux éminemment hydraulique du XIXè siècle. Elle est produite en Midi-Pyrénées (usine d'Izaourt dans les Hautes Pyrénées). Elle est essentiellement composée de chaux, de calcite et de silicate C2S (tableau 1).

\begin{tabular}{|l|l|l|l|l|l|l|}
\hline $\begin{array}{l}\text { Chaux } \\
\mathrm{Ca}(\mathrm{OH}) 2\end{array}$ & $\begin{array}{l}\text { Calcite } \\
\mathrm{CaCO} 3\end{array}$ & $\mathrm{C} 2 \mathrm{~S}$ & $\begin{array}{l}\text { Autres } \\
\text { silicates }\end{array}$ & aluminates & $\begin{array}{l}\text { Quartz et } \\
\text { divers }\end{array}$ & Eau libre \\
\hline 17.6 & 30.4 & 40.5 & 0 & 0 & 9.5 & 2 \\
\hline
\end{tabular}

Tableau 1. Composition minéralogique de la chaux SOCLI NHL 3.5, en pourcentage.

Nous avons observé, pour les trois matériaux (brique, mortier de chaux et pierre calcaire) testés en compression axiale, une phase

élastique linéaire, jusqu'à 40 à $50 \%$ de la charge de rupture selon les matériaux, suivie d'une première phase d'écrouissage linéaire conduisant à la ruine (figure 3a). La résistance à la compression $f_{c}$ des 
briques, perpendiculairement à leur plan, est 13.8MPa (écart type 2.4MPa) et leur module d'Young, de $5500 \mathrm{MPa}$ (écart type $1400 \mathrm{MPa}$ ). Elles sont orthotropes et leurs caractéristiques mécaniques dans leur propre plan sont plus élevées (résistance $f_{c}$ deux fois plus forte, module multiplié par 3 ).

Les caractéristiques du mortier à l'âge d'un mois et deux mois sont : $f_{c}=3.7 \mathrm{MPa}$ (écart type 0.3MPa), $\mathrm{E}=5200 \mathrm{MPa}$ (écart type 900MPa). Remarquons que briques et mortier, au vu de ces résultats, ont eu quasiment la même déformabilité en phase travaux, et, en particulier, au moment du décintrement. Notre étude a montré que, du fait du vieillissement (simulé en laboratoire par carbonatation accélérée), la résistance du mortier, à long terme, atteint 2.2 à 2.7 fois sa résistance à un mois. Le module du mortier augmente jusqu'à $7800 \mathrm{MPa}$. La loi de comportement du mortier vieilli artificiellement par carbonatation est donnée sur la figure $3 \mathrm{~b}$.

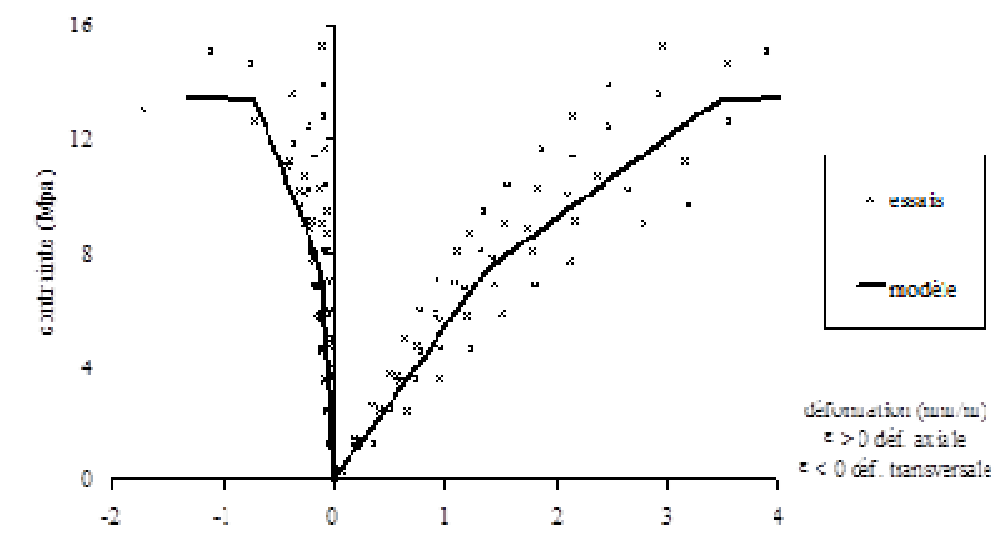

Figure 3a. Essais et modélisation des briques.

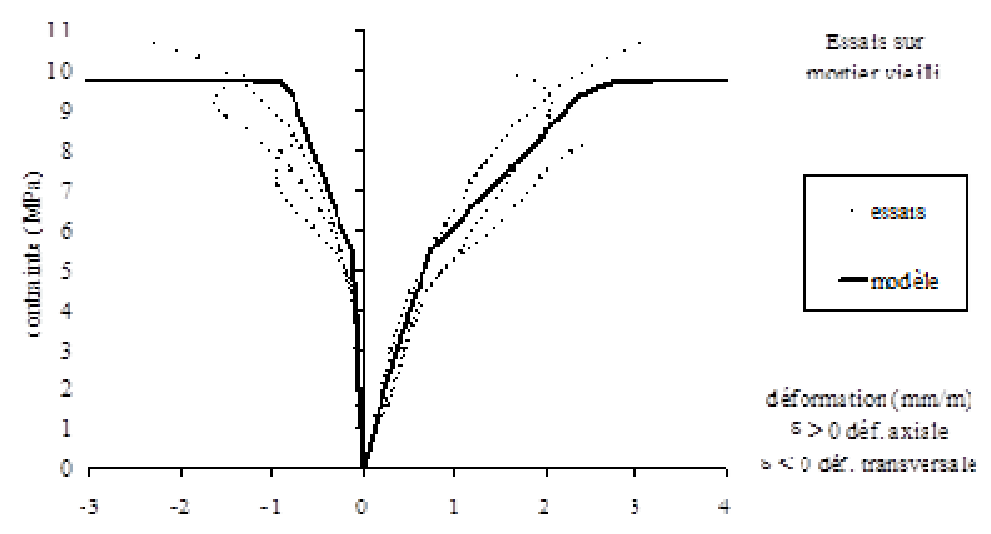

Figure 3b. Essais et modélisation du mortier de chaux.

Ainsi, aujourd'hui, phénomènes de corrosion et d'altération mis à part, briques et joints ont une résistance similaire, mais, contrairement aux idées couramment admises, c'est le mortier qui est le plus rigide. La pierre, quant à elle, a une résistance de 50MPa et un module de $28000 \mathrm{MPa}$, ce qui en fait, et de très loin, le matériau le plus rigide du pont étudié.

\subsection{Etude expérimentale de la maçonnerie de briques}

La maçonnerie est un ensemble de blocs (de briques) et de joints (de mortier de chaux hydraulique). C'est donc un composite. Nous avons mis en œuvre une méthode numérique qui nous a permis de construire un modèle homogénéisé intégrant la phase élastique et la phase d'écrouissage jusqu'à rupture. Les modèles élasto-plastiques élémentaires correspondant à chacun des deux matériaux sont présentés sur les figures 3 . Le modèle résultant a été comparé aux courbes obtenues lors d'une 
campagne d'essai de rupture par compression de petits éléments de maçonnerie. Une première série d'essais sur des éléments dits " sandwiches " constitués simplement d'un empilement alterné de joints et de briques permet de valider un premier niveau d'homogénéisation de type "série ". En un second temps, des " murets ", incluant des joints verticaux dus au montage en quinconce des briques, permet de valider un modèle d'homogénéisation d'un volume élémentaire représentatif de maçonnerie.

\subsubsection{Procédure expérimentale}

Les modèles expérimentaux en maçonnerie, construits au laboratoire, sont donc de deux types : les « sandwiches », qui ne comportent que des joints de mortier horizontaux, et les «murets » qui comportent des joints horizontaux et verticaux. On notera que la maçonnerie de brique construite en région Sud Ouest de la France comporte des joints épais, de 1 à $2 \mathrm{~cm}$ d'épaisseur. Les dimensions transversales des sandwiches sont représentées sur la figure 4. L'épaisseur des joints de mortier est de $2 \mathrm{~cm}$. L'instrumentation des éprouvettes, soit dix jauges et deux capteurs de déplacement, est visible sur le schéma de la figure 4.
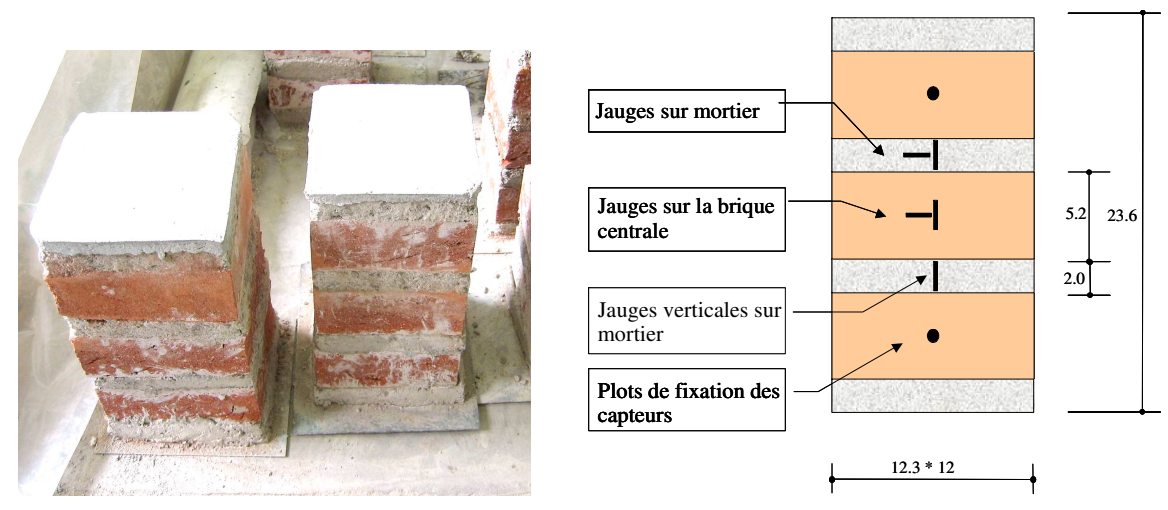

Figure 4. Géométrie des "sandwiches"

Les «murets » constituent un volume représentatif de la maçonnerie réelle, du point de vue de son comportement mécanique. Une étude numérique préalable a été nécessaire pour définir la géométrie des murets. Son but était de définir le nombre de briques à mettre en place horizontalement et verticalement de façon à obtenir un "Volume Elémentaire Représentatif " c'est-à-dire dont le comportement ne dépend pas de sa taille. Les murets testés sont composés de neuf épaisseurs de briques (figure 5) et ont une hauteur de $61.8 \mathrm{~cm}$ (et une masse d'environ $110 \mathrm{~kg}$ ). Ils sont équipés de 4 capteurs de déplacement et de 10 jauges. Les essais ont eu lieu deux mois après leur fabrication.
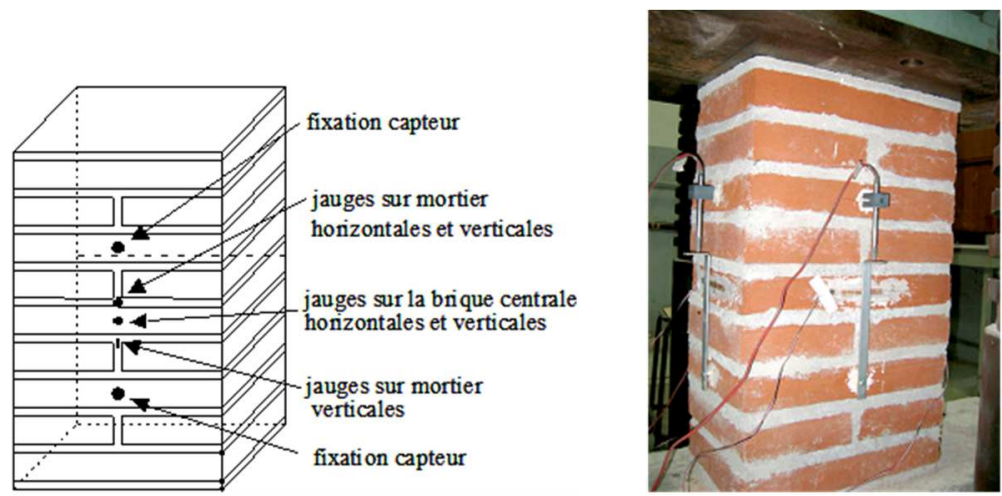

Figure 5. Géométrie et instrumentation des " murets » 


\subsubsection{Essais sur sandwiches}

Pendant les essais, on a pu observer une rupture des échantillons sans glissement relatif entre les matériaux. Le comportement est quasi linéaire jusqu'à environ $40 \%$ de la contrainte de rupture. Le raccourcissement enregistré par les capteurs permet de calculer un module moyen du composite brique / mortier avec joints horizontaux, qui s'élève seulement à $5200 \mathrm{MPa}$ (tableau 2).

\begin{tabular}{|l|l|l|l|}
\hline $\mathrm{E}(\mathrm{MPa})$ & Sandwich & Mortier & Brique $\mathrm{E}_{3}$ \\
\hline Moyenne sur les deux mois & 5254 & 5204 & 5524 \\
\hline
\end{tabular}

Tableau 2. Module moyen des sandwiches. Comparaison avec les matériaux de base.

Le mortier des joints conserve un comportement linéaire jusqu'à sa propre résistance à la compression. La plastification du mortier n'entraîne pas la rupture du sandwich, qui s'effondre lorsque la brique cède. Rappelons ici que, dans la direction transversale, la brique est trois fois plus rigide que le mortier. L'adhérence entre les deux matériaux, observée lors des essais, crée un effet de confinement du mortier qui, dans un premier temps, lui procure un écrouissage positif et retarde sa rupture. Dans un second temps, les grandes déformations transversales du mortier déclenchent la rupture prématurée des briques. L'adhérence entre les deux matériaux a donc un rôle fondamental dans la résistance du composite. Cette adhérence est améliorée par la rugosité des briques, moulées à l'ancienne avec du sable, et le fait qu'elles aient été immergées avant montage des éléments.

\subsubsection{Essais sur murets}

Les éléments testés comportent des joints horizontaux et un joint vertical (figure 5). Trois murets ont été réalisés. Nous donnons ici les résultats les plus significatifs. La fissuration d'un muret s'est développée sans glissement apparent entre les matériaux mortier et brique. La résistance à la compression du muret testé fut : 10.2 $\mathrm{MPa}$. Le tableau 3 permet la comparaison avec les autres essais.

\begin{tabular}{|l|l|l|l|l|l|}
\hline & muret & sandwiches & $\begin{array}{l}\text { mortier } \\
(4 * 4 * 16)\end{array}$ & $\begin{array}{l}\text { Mortier (cylindres } \\
6 * 12)\end{array}$ & brique \\
\hline $\mathrm{f}_{\mathrm{c}}(\mathrm{MPa})$ & 10.2 & 9,0 & 3.9 & 3.5 & 13.8 \\
\hline
\end{tabular}

Tableau 3. Résistance des murets, sandwiches, mortier et brique, à 2 mois.

\subsubsection{Récapitulatif des résultats des essais}

Le raccourcissement entre les capteurs permet de calculer le module moyen indiqué dans le tableau 4. La valeur mesurée est légèrement plus faible que celle de chacun des composants. Le comportement des briques reste quasi linéaire jusqu'à environ $7 \mathrm{MPa}$, soit environ $70 \%$ de la résistance du muret.

\begin{tabular}{|l|l|l|l|l|}
\hline & muret & sandwiches & Mortier & Brique \\
\hline E (MPa) & 4311 & 5254 & 5204 & 5524 \\
\hline Ecart type & & 539 & 901 & 1415 \\
\hline
\end{tabular}

Tableau 4. Module moyen du muret, des sandwiches, du mortier et de la brique. 


\subsection{Modélisation de la maçonnerie}

Il s'agit ici de choisir le modèle le plus performant vis à vis des résultats des essais effectués au laboratoire sur les éléments composites, sandwiches et murets. Les trois modèles suivants ont été envisagés : modèle élasto-plastique avec écrouissage, et critère de plasticité de type Von Mises (modèle 1), modèle élasto-plastique sans écrouissage, et critère de plasticité de type Drucker-Prager (modèle 2), modèle élastique endommageable type Mazars (modèle 3).

La démarche adoptée pour sélectionner le modèle s'est opérée en deux phases. Dans un premier temps, les paramètres théoriques ont été ajustés en conformité avec les résultats d'essais effectués sur les matériaux de base, brique, mortier, individuellement. Dans un deuxième temps, les lois de comportement paramétrées ont été intégrées à un calcul par élément fini reproduisant numériquement les essais de compression sur les sandwiches et les murets. Le choix du modèle théorique parmi les trois modèles retenus, s'est fait sur la comparaison des courbes contraintesdéformations des composites, obtenues par simulations numériques, d'une part, et par les essais réels, d'autre part (essais à 2 mois).

La différence de performance des trois modèles n'apparaît bien entendu que dans le domaine non linéaire, et donc au-delà de $3 \mathrm{MPa}$ (figures 6 et 7). Le modèle 1, modèle élasto-plastique avec une loi d'évolution contraintes-déformations trilinéaire, a été adopté. Les paramètres sont résumés dans le tableau 5.

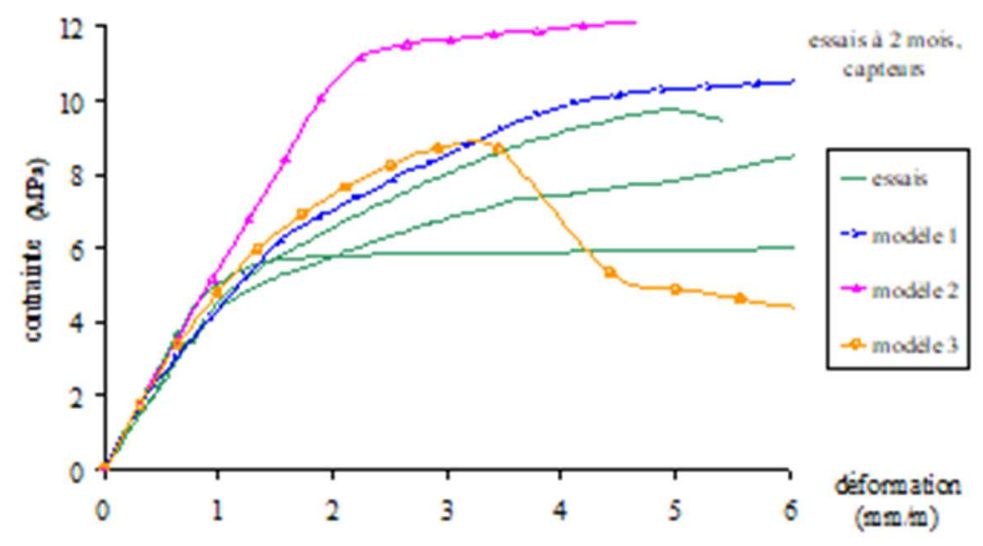

Figure 6. Modélisation du comportement des sandwiches à 2 mois.

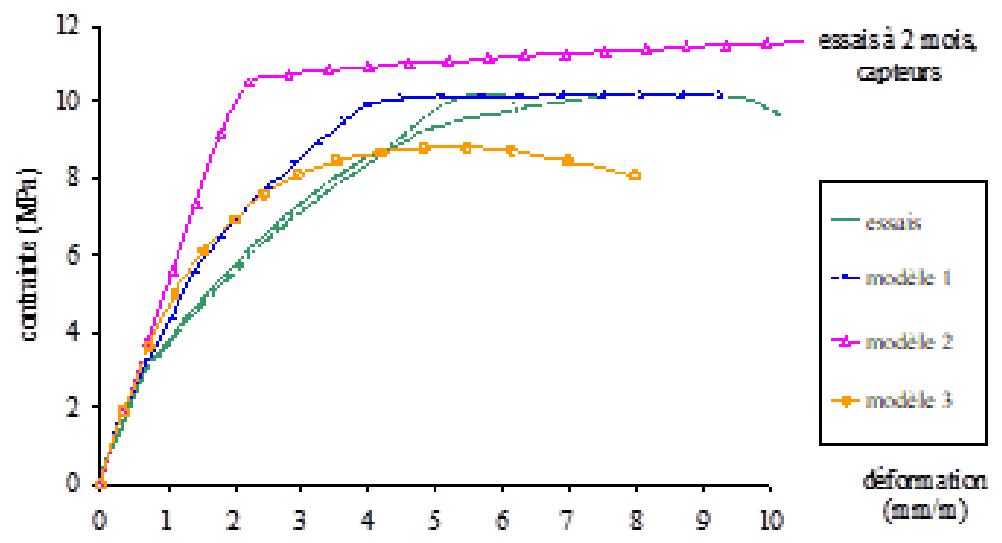

Figure 7. Modélisation du comportement des murets à 2 mois. 
La modélisation proposée, pour tous les matériaux en présence comprend une partie élastique linéaire jusqu'à $40 \%$ à $50 \%$ de la contrainte de rupture selon les matériaux (figure 8 ), suivie d'une phase d'écrouissage rectiligne jusqu'à un palier d'écoulement horizontal. Le critère de plasticité associé est le critère de Von mises. Les modèles théoriques proposés prédisent la charge de ruine des maçonneries à partir de la loi de comportement de ses composants, ce qui valide la procédure d'homogénéisation utilisée pour calculer la loi de comportement d'un Volume Elémentaire Représentatif d'une maçonnerie. Celle-ci est à son tour approchée par une loi élasto-plastique dont les caractéristiques sont données dans le tableau 5. La procédure d'homogénéisation est également utilisée pour estimer la loi de comportement des murs tympans en moellons et du remplissage en moellons bruts (figure 9).

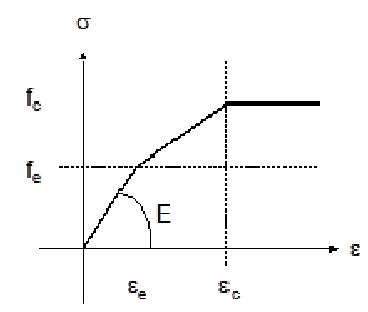

Figure 8. Modèle 1 : modèle élasto-plastique, avec critère de plasticité de Von Mises.

\begin{tabular}{|l|l|l|l|l|}
\hline & $\begin{array}{l}\text { Voûte en } \\
\text { briques }\end{array}$ & $\begin{array}{l}\text { Bandeau et } \\
\text { sommier en pierre }\end{array}$ & $\begin{array}{l}\text { Murs tympans en } \\
\text { moellons }\end{array}$ & $\begin{array}{l}\text { Remplissage en } \\
\text { moellons bruts }\end{array}$ \\
\hline$E(\mathrm{MPa})$ & 6800 & 28100 & 21700 & 21700 \\
\hline$V$ & 0.13 & 0.25 & 0.22 & 0.22 \\
\hline $\mathrm{f}_{\mathrm{e}} / \mathrm{f}_{\mathrm{c}}$ & 0.5 & 0.4 & 0.4 & 0.4 \\
\hline $\mathrm{f}_{\mathrm{c}}(\mathrm{MPa})$ & 12 & 49 & 42 & 42 \\
\hline$\varepsilon_{\mathrm{c}}(\mathrm{mm} / \mathrm{m})$ & 3 & 2.5 & 3 & 3 \\
\hline
\end{tabular}

Tableau 5. Caractéristiques mécaniques des maçonneries du pont étudié

Au vu des résultats numériques, la voûte en brique apparaît nettement comme l'élément le plus faible du pont, et non pas le remplissage, comme on aurait pu s'y attendre. En effet, elle a un module et une résistance à la compression quatre fois plus faible que les autres parties de l'ouvrage. Le bandeau est l'élément le plus rigide. 


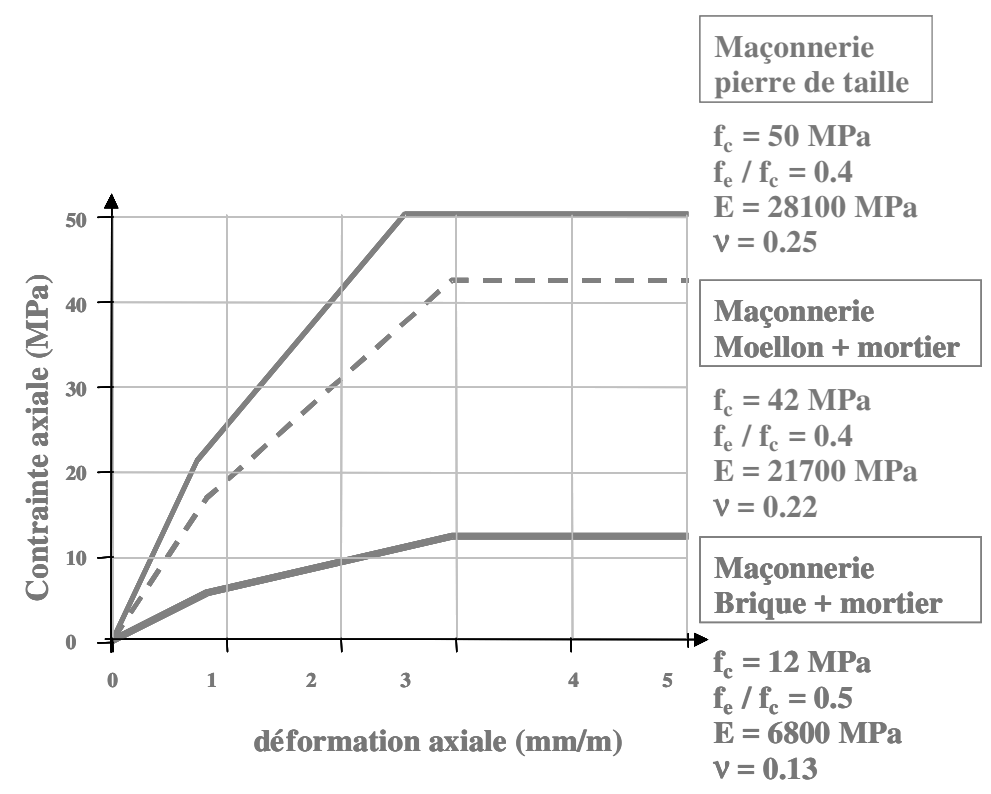

Figure 9. Comportement mécanique des maçonneries jusqu'à rupture.

\subsection{Eléments joints}

Le comportement théorique décrit précédemment et associé aux éléments finis massifs est, dans le code de calcul que nous utilisons, identique en traction et en compression. Ceci ne correspond pas à la réalité, puisque la résistance à la traction observée expérimentalement est très faible. C'est la raison pour laquelle, dans notre modèle numérique, les phénomènes de fissuration par traction et cisaillement des joints de mortier sont concentrés dans des éléments joints prévus à cet effet. Ils représentent le comportement de la surface de contact entre le mortier et la brique. Les éléments joints ont un comportement différent en traction et en compression (figure 10). Leur critère de plasticité est de type Coulomb.

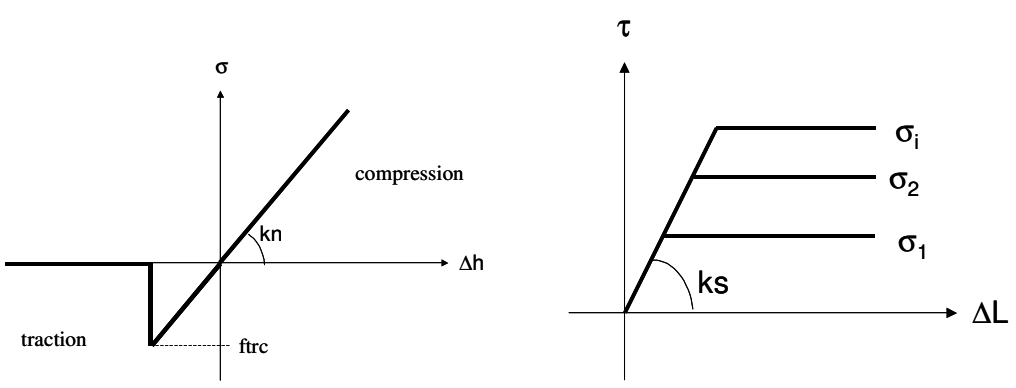

Figure 10. Comportement des éléments joints.

Les valeurs des paramètres du modèle de Coulomb (tableau 6) ont été établies par des essais réalisés à l'aide de la boite de Casagrande. Les échantillons utilisés pour ces essais sont formés d'un cylindre de brique (obtenu par carottage) surmonté d'une couche de mortier coulé sur la brique (figure 11).

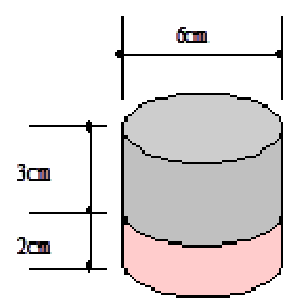

Figure 11. Dimensions des éprouvettes utilisées pour caractériser le modèle de Coulomb. 


\begin{tabular}{|l|l|l|l|l|}
\hline Cohésion & $\begin{array}{l}\text { Angle de } \\
\text { frottement interne }\end{array}$ & $\begin{array}{l}\text { Résistance à la } \\
\text { traction }\end{array}$ & $\begin{array}{l}\text { Raideur normale à } \\
\text { l'interface }\end{array}$ & $\begin{array}{l}\text { Raideur tangentielle à } \\
\text { l'interface }\end{array}$ \\
\hline $0.1 \mathrm{MPa}$ & $49^{\circ}$ & $\mathrm{ftrc}=0.6 \mathrm{MPa}$ & $\mathrm{k}_{\mathrm{n}}=10^{7} \mathrm{MPa}$ & $\mathrm{k}_{\mathrm{s}}=10^{5} \mathrm{MPa}$ \\
\hline
\end{tabular}

Tableau 6. Caractéristiques de l'interface brique - mortier.

\section{PHASE 3 : MODELISATION ET CALCUL D'UN PONT}

\subsection{Principe de la modélisation}

Principes fondamentaux du modèle numérique :

- Le maillage du pont est automatisé (pour une application ultérieure) et ne nécessite qu'un minimum de paramètres donnés par l'utilisateur (niveau du centre du cercle décrivant l'intrados, niveau de fondation, niveau supérieur des murs, rayon de l'intrados et son angle d'ouverture, épaisseur du bandeau en clé et de la voûte en clé, hauteur du sommier, dimensions des culées, épaisseur du mur et des bandeaux, et, enfin, largeur de la voûte en brique).

- Le modèle du pont est constitué de 4 modèles de maçonneries différentes (figure 12) : celles de la voûte, des bandeaux et des sommiers, des murs et des culées, du remplissage. A chacun des modèles est affecté une des lois de comportement homogénéisées présentées dans le chapitre précédent.

- Le maillage des maçonneries est constitué d'éléments finis massifs tridimensionnels supportant les quatre modèles décrits précédemment, entre lesquels s'interposent (de façon non systématique) des modèles de joints rayonnants qui découpent le pont en voussoirs. Ceux-ci peuvent se séparer les uns des autres par glissement et/ou décollement selon le critère de Coulomb décrit ci-dessus (figure 13). Les joints traversent la voûte et le remplissage.

- Le pont peut glisser et se décoller par rapport au sol de fondation par l'intermédiaire d'un modèle de joints affectés d'une loi de Coulomb et positionnés entre la pile et la fondation. La butée des culées sur les remblais est négligée, par sécurité.

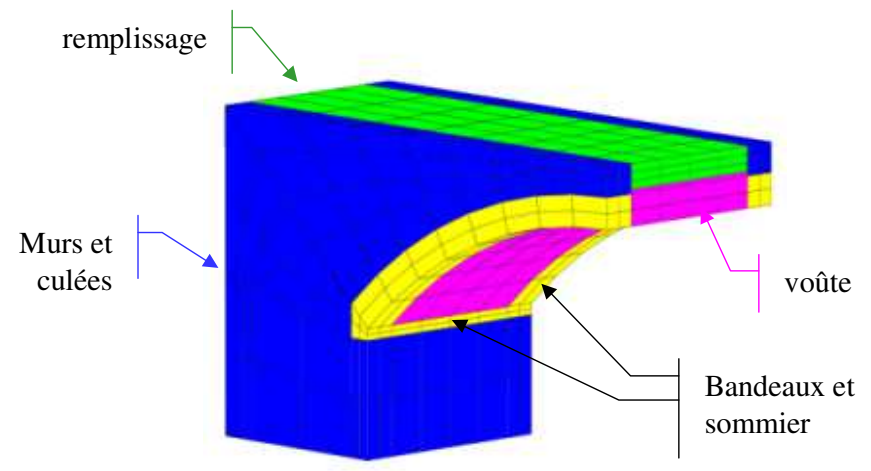

Figure 12. Modélisation du pont. Les quatre maçonneries en présence. 


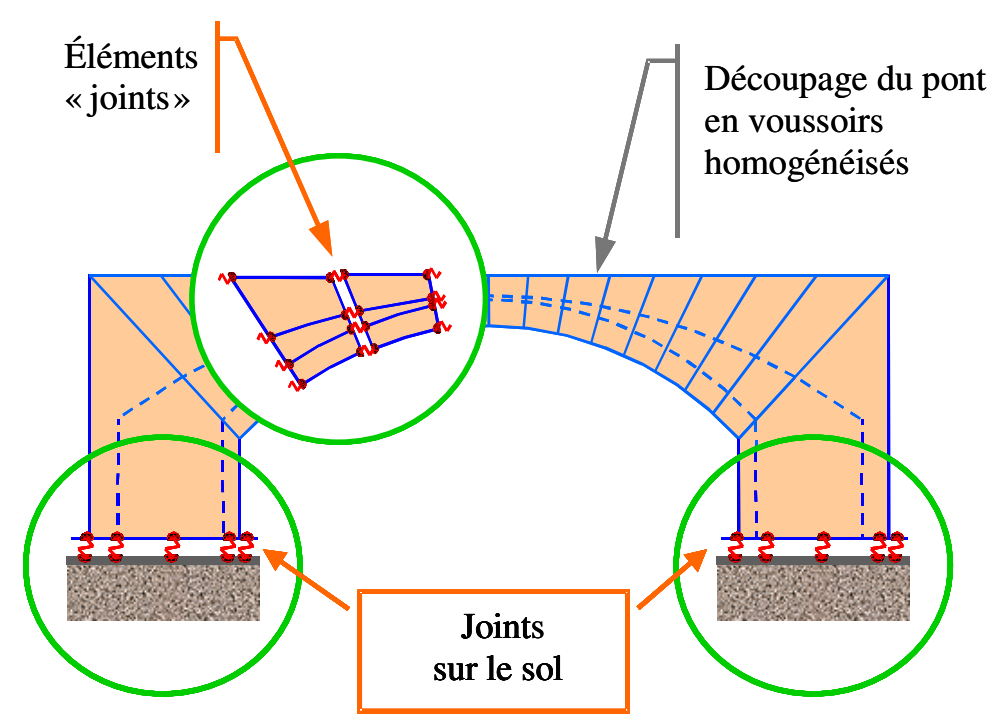

Figure 13. Découpage du pont en voussoirs. Position des joints de Coulomb.

Nous avons choisi de soumettre l'ouvrage à son poids propre (10MN) ainsi qu'au poids d'un véhicule non centré longitudinalement (figure 14). Il est constitué d'un tandem d'essieux, dont la géométrie est conforme au type TS de l'EUROCODE 1 (2 essieux de 300kN en service). Le positionnement transversal de ce tandem est paramétré. Les charges concentrées sont appliquées sous la forme d'un déplacement vertical imposé des points d'impact des roues, déplacement augmenté jusqu'à rupture du pont.

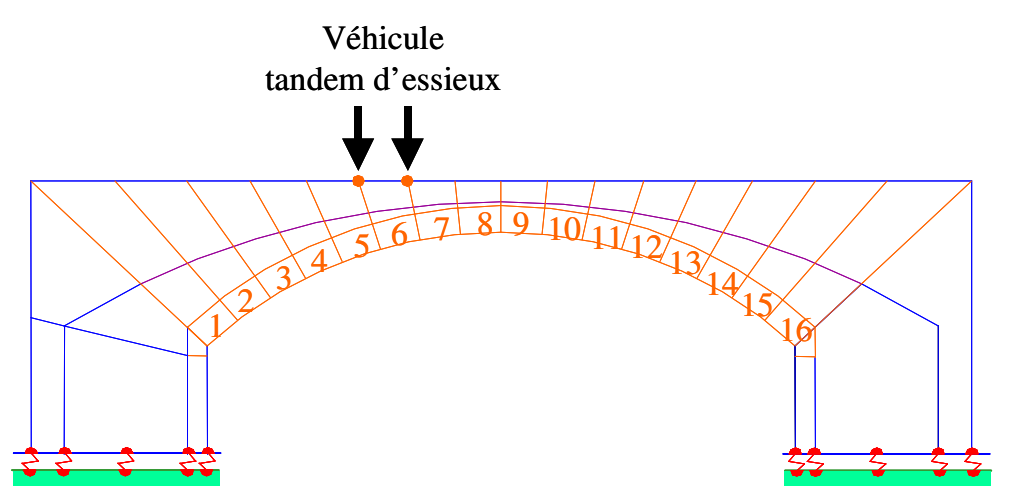

Figure 14. Position des charges sur le pont.

\subsection{Résultats et analyse}

L'analyse des résultats du calcul a dégagé des informations essentielles sur la circulation des efforts internes et sur le mécanisme de ruine de l'ouvrage. Il apparaît que celui-ci est associé au glissement des fondations mis en évidence par le tracé de la déformée du pont (figure 15). Du fait de ce glissement, les joints de Coulomb, qui rayonnent à partir de l'axe de la voûte, s'ouvrent, entraînant la ruine du pont avec des déplacements importants des culées. L'ouverture des joints est très faible en service normal, de l'ordre du centième de millimètre, et atteint $1 \mathrm{~mm}$ au moment du glissement de la fondation. 


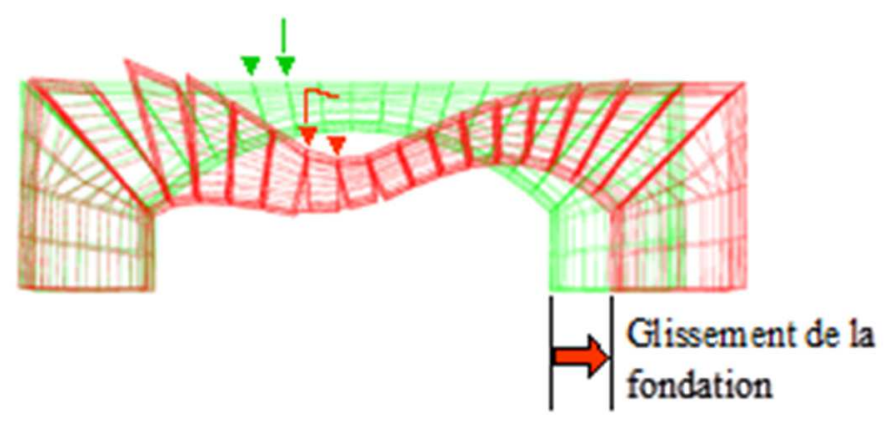

Figure 15. Allure de la déformée du pont à la rupture.

On constate que les zones les plus comprimées du pont sont, d'une part, les bandeaux, et d'autre part, les angles extérieurs des sommiers. La figure 16 montre l'intensité des contraintes de compression au moment de la ruine du pont ; celles ci restent modérées par rapport à la résistance à la compression des matériaux, ce qui signifie que la ruine est principalement due à l'ouverture des joints.

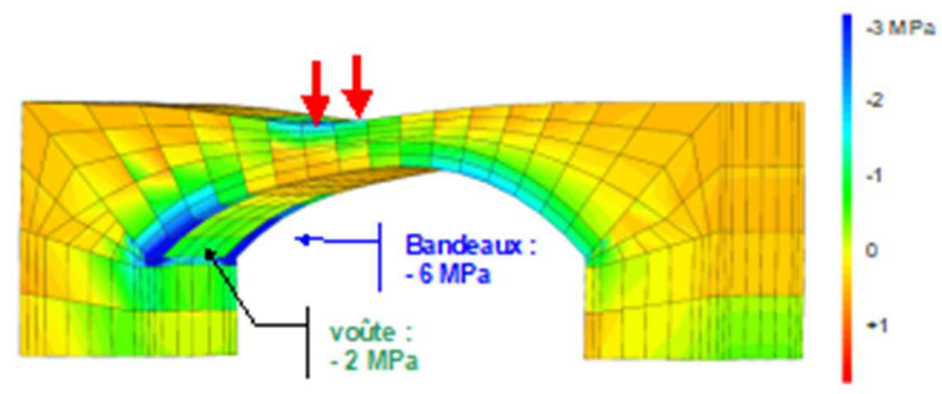

Figure 16. Contraintes de compressions à la ruine du pont.

On peut également noter le gradient des contraintes de compression entre les bandeaux et la voûte, à l'origine de cisaillements à l'interface entre ces deux zones, ainsi que l'apparition de zones tendues, transversalement, à l'intrados de la voûte; ces deux derniers phénomènes peuvent conduire à l'apparition de fissures longitudinales sous le pont. Elles correspondent à une pathologie souvent observée par les maîtres d'ouvrages. Dans le plan de contact entre l'ensemble voûte / remplissage d'une part, et l'ensemble bandeau / murs d'autre part, les cisaillements et les tractions sont concomitants, ce qui augmente le risque de rupture localisée. Selon nos calculs, ce phénomène transversal s'amplifie lorsque la position du véhicule est désaxée transversalement vers l'extérieur de la voie. Par conséquent, l'élargissement des ponts apparaît comme un facteur aggravant de cette pathologie.

La figure 17 montre la loi de comportement du pont pour deux types de conditions aux limites (appuis bloqués ou glissant sur la fondation). Le processus de ruine par ouverture des joints transversaux visualisé sur la figure 15 correspond aux appuis glissants. Si les appuis sont bloqués la capacité portante du pont est augmentée jusqu'à l'atteinte des fissures longitudinales ou bien jusqu'à la rupture en compression d'un élément de la maçonnerie. 


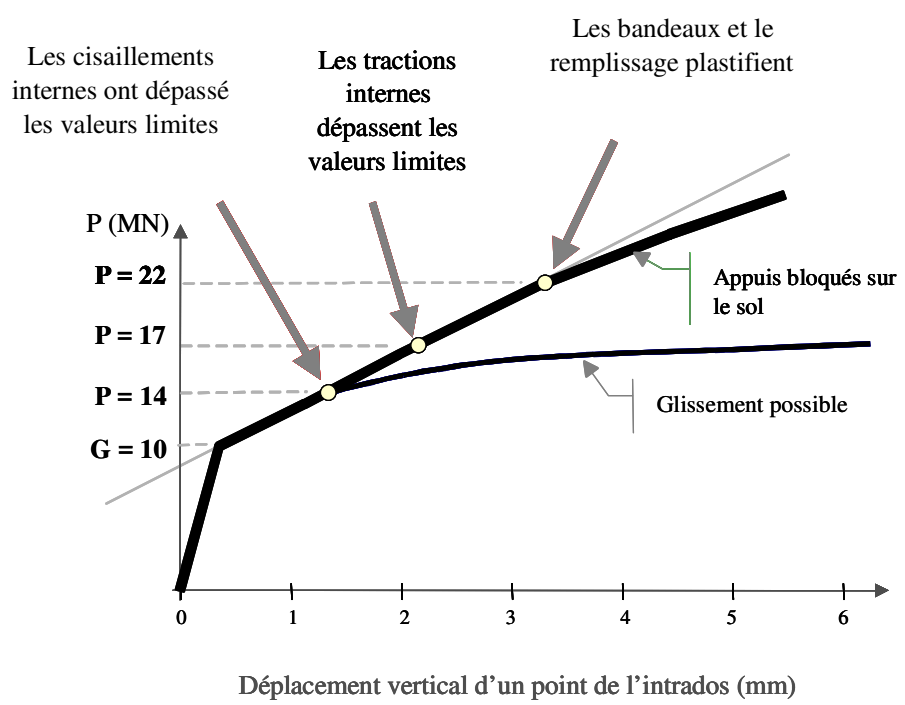

Figure 17. Force appliquée sur le pont en fonction du déplacement vertical dans la zone d'application, pour deux configurations de conditions aux limites avec le sol (Appuis bloqués ou glissement possible suivant le critère de Coulomb)

\section{BILAN ET PERSPECTIVES}

La méthode de requalification par le calcul que nous venons de décrire pourrait s'appliquer à la plupart des ouvrages en maçonnerie. Cette méthode en trois phases permet de décrire les phénomènes internes des différentes parties de l'ouvrage, en service et jusqu'à la rupture, dans les trois dimensions du pont. Elle prend en compte la phase non linéaire des matériaux constitutifs et la fissuration transversale. Le modèle numérique mis en œuvre n'exclut a priori aucun phénomène de ruine ; il est alors applicable à tout type d'ouvrage maçonné et constitue de ce fait un fil conducteur intéressant pour la requalification des ouvrages anciens.

\section{REMERCIEMENTS}

Cette recherche a vu le jour grâce à l'aimable participation de la Société Nationale des Chemins de Fer français, et de Réseau Ferré de France. Nous remercions tout spécialement Monsieur Bernard PLU pour l'intérêt qu'il manifeste à l'égard de ces travaux. Nous remercions également le Commissariat à l'Energie Atomique pour la mise à disposition de son outil de calcul aux éléments finis, CASTEM, dans sa version «Education et recherches".

\section{BIBLIOGRAPHIE}

Cavicchi A., Gambarotta L., "Collapse analysis of masonry bridges taking into account arch-fill interaction", Engineering Structures, 27, 2005. pp. 605-615.

Cecchi A., Sab K., "A multi-parameter homogenization study for modelling elastic masonry", European Journal of Mechanics, A/ Solids 21, 2002. pp. 249-268.

Combescure D., Modélisation du comportement sous chargement sismique des structures de bâtiment comportant des murs de remplissage en maçonnerie, thèse de doctorat, Ecole Centrale de Paris : 1996, $189 \mathrm{p}$.

Cruz-diaz Jl., Sellier A., Capra B., Delmotte P., Mebarki A., « Fiabilité des murs de contreventement en maçonnerie. Calibration des coefficients partiels d'un modèle simplifié », REGC, vol 6/3 - 2002 - pp.447456. 
Davey J., Augarde C., "Modelling skewed masonry arches", Structural Engineer, vol.79, n7, 3 april 2001, pp.14-15.

Fanning P.J., Boothby T.E., Roberts B.J., "Longitudinal and transverse effects in masonry arch assessment", Construction and building Materials, vol.15, Issue 1, february. 2001, pp. 51-60.

Fanning P.J., Salomoni V-A., Sloan D-T., "Non linear modelling of a multi-span arch bridge under service load conditions", 10th international conference and exhibition, "Extending the life of bridges", London, UK, 1-3, july 2003.

Gilbert M., Ahmed H.M., Sollis A.J., Masonry arch bridge analysis software RING, University of Sheffield, 2005, http://www.ring.shef.ac.uk.

Giordano A., Mele E., De Luca A., "Modelling of historical masonry structures : comparison of different approaches through a case study", Engineering Structures, vol.24, issue 8, august 2002, pp. 10571069.

Lee J-S., Pande G-N., Kral J-B., "Etude comparative de la simulation numérique d'ouvrages en maçonnerie", Materials and structures, 31, n²11, GBR, 2001, pp. 473-479.

Rosson B.T., Soyland K., Boothby T.E., "Inelastic behaviour of sand-lime mortar joint masonry arches", Engineering Structures, vol.20, n¹-2, 1998, pp. 14-24.

Sab K., Yield design of thin periodic plates by a homogenization technique and an application to masonry walls, Académie des sciences, C.R. Mécanique 331, 2003, pp. 641-646.

Séjourné P. Grandes voûtes, Bourges : imprimerie Vve Tardy, 1913, 6 tomes.

SETRA / LCPC (1982) Les ponts en maçonnerie, historique, stabilité, utilisation du programme VOUTE.

Thavalingham A., Bicanic N., Robinson J-I., Ponniah D-A., "Computational framework for discontinuous modelling of masonry arch bridges", Computers \& Structures vol. 79, Issue 19, July 2001, pp. 18211830.

Zucchini A., Lourenco P.B., "A micro-mechanical model for the homogenisation of masonry", International journal of solids and structures 39, February 2002, pp. 3233-3255. 